\title{
Nonlinear Optics in Silicon Core Fibers
}

\author{
A.C.Peacock ${ }^{1}$, P.Mehta ${ }^{1}$, T.D.Day ${ }^{2}$, J.R.Sparks ${ }^{2}$, J.V.Badding ${ }^{2}$ and N.Healy ${ }^{1}$ \\ 1. Optoelectronics Research Centre, University of Southampton, Southampton SO17 1BJ, UK \\ 2. Department of Chemistry and Materials Research Institute, Pennsylvania State University 16802 PA, USA
}

\begin{abstract}
The nonlinear transmission properties of hydrogenated amorphous silicon core fibers are characterized for short pulse propagation. Simple pump-probe experiments are employed to demonstrate their use for all-optical control via phase and amplitude modulation.
\end{abstract}

\section{Introduction}

Silicon waveguides are becoming an increasingly popular platform for nonlinear optical signal processing. The incorporation of silicon into the optical fiber geometry provides an important step towards integrating semiconductor functionality with conventional fiber infrastructures as well as allowing for the construction of robust devices with novel waveguiding properties. In this paper we review our progress in characterizing the nonlinear transmission properties of the silicon core fibers that are fabricated using a high pressure chemical deposition technique [1]. Although typical core sizes for these fibers are of the order of a few micron, the high nonlinear index of the hydrogenated amorphous silicon core material has enabled the observation of substantial spectral broadening and allowed for all-optical control using both cross-phase modulation (XPM) and cross-absorption modulation (XAM) [2].

\section{Nonlinear Characterization}

The fibers are fabricated using our deposition technique in which hydrogenated amorphous silicon (a-Si:H) is infiltrated into the pore of a silica capillary. a-Si:H is emerging as an important material for nonlinear photonics owing to its high nonlinearity and low losses. Fig. 1(a) shows the spectral evolution in a $5.7 \mu \mathrm{m}$ diameter core fiber clearly showing nonlinear broadening due to self-phase modulation with $\sim 100 \mathrm{~nm}$ bandwidth. Nonlinear characterization reveals the two-photon and free-carrier absorption parameters as $\beta$ TPA $\sim 0.8 \mathrm{~cm} / \mathrm{GW}$ and $\sigma \sim 1 \times 10^{-16} \mathrm{~cm}^{2}$, respectively, with a Kerr coefficient of $n_{2} \sim 1.5 \times 10^{-13} \mathrm{~cm}^{2} / \mathrm{W}$ which is greater than twice that of crystalline silicon. As a means of exploiting the large nonlinear parameters to demonstrate device functionality, a series of simple pump-probe experiments have been conducted. Fig. 1(b) shows the results of XPM induced by a strong pump at $1.54 \mu \mathrm{m}$ acting on a weak probe at $1.59 \mu \mathrm{m}$, as a function of the delay between the pulses at the fiber input. From this it is clear that when the probe propagates in the presence of the pump it experiences strong spectral modulation. Fig. 1(c) then shows the effects of XAM on a probe at $1.57 \mu \mathrm{m}$ due to a $\sim 750 \mathrm{fs}$ pump at $1.54 \mu \mathrm{m}$. The results illustrate the ultra-fast response due to TPA, followed by a rapid recovery via carrier relaxation and then the slower recombination due to the free carriers. The device performance will be discussed in relation to the material quality and core size.
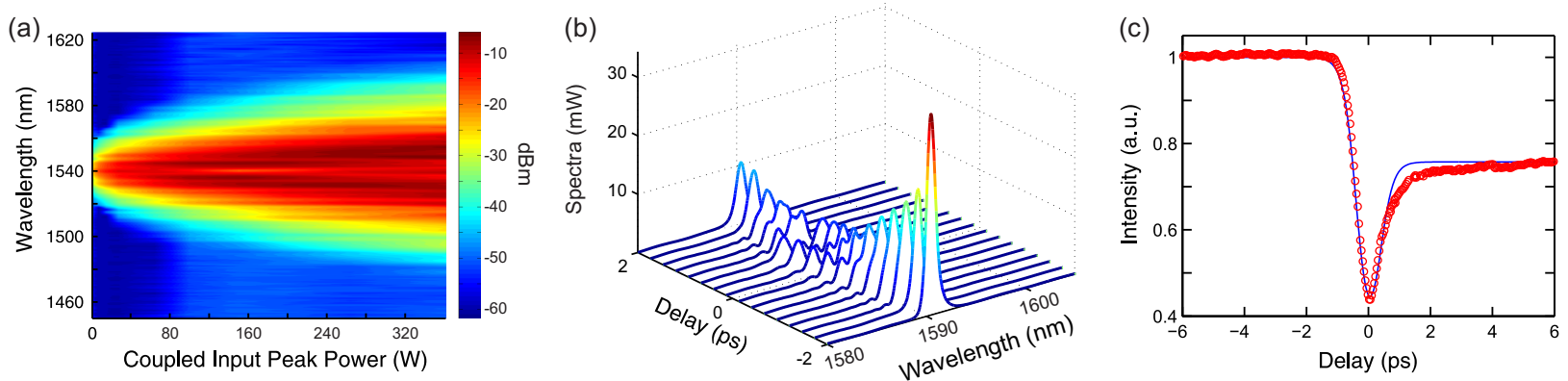

Fig. 1 (a) SPM as a function of input power (b) XPM and (c) XAM as functions of delay

\section{References}

1. P. Mehta, N. Healy, N. F. Baril, P. J. A. Sazio, J. V. Badding, and A. C. Peacock, "Nonlinear transmission properties of hydrogenated amorphous silicon core optical fibers," Opt. Express 16, 16826-16831 (2010).

2. P. Mehta, N. Healy, T. D. Day, J. R. Sparks, P. J. A. Sazio, J. V. Badding, and A. C. Peacock, "All-optical modulation using two-photon absorption in silicon core optical fibers," Opt. Express 19, 19078-19083 (2011). 